[Chem. Pharm. Bull.

34( 8$) 3284-3289(1986)]$

\title{
3,4-Seco-lupane Type Triterpene Glycosyl Esters from a Korean Medicinal Plant, Acanthopanax chiisanensis (Araliaceae)
}

\author{
Ryoji Kasai, ${ }^{a}$ Kazuhiro Matsumoto, ${ }^{a}$ Shigenori Taniyasu, ${ }^{a}$ \\ Osamu TanaKa, ${ }^{*, a}$ JeUng-HeE Kim, ${ }^{b}$ \\ and DUG-RYONG HAHN ${ }^{b}$ \\ Institute of Pharmaceutical Sciences, Hiroshima University School of Medicine, ${ }^{a}$ \\ Kasumi, Minami-ku, Hiroshima 734, Japan and Colkege of Pharmacy, \\ Chung-Ang University, ${ }^{b} 221$ Huksuk-Dong, \\ Kwanak-ku, Seoul 151, Korea
}

(Received February 3, 1986)

\begin{abstract}
The experimental details of the isolation and structural determination of chiisanoside (1), a new 3,4-seco-lupane type triterpene glycosyl ester, from leaves and stem bark of Acanthopanax chiisanensis are described. From leaves of this plant, two homologous glycosyl esters, named isochiisanoside and its methyl ester ( 2 and 3 ), were also isolated. Their structures were elucidated on the basis of chemical and spectral data and confirmed by the derivation of these compound from 1 .
\end{abstract}

Keywords-Acanthopanax chiisanensis; Araliaceae; chiisanoside; isochiisanoside; chiisanogenin; anhydro-chiisanogenoic acid; 3,4-secotriterpenoid; lupane derivative; oligo-glycosyl ester

A folk medicine, leaves and stem-bark of Acanthopanax chiisanensis NAKAI ( 智異山五加, Araliaceae), is used as an anti-rheumatic, an anti-inflammatory and a tonic in Korea. From this folk medicine, a new glycosyl ester (1) named chiisanoside was isolated and identified as the $\alpha$ L-rhamnopyranosyl $(1 \rightarrow 4)$ - $\beta$-D-glucopyranosyl $(1 \rightarrow 6)$ - $\beta$-D-glucopyranoside of $(1 R)-1,11 \alpha$ dihydroxy-3,4-seco-lupa-4(23),20(29)-diene-3,28-dioic acid 3,11-lactone (Chart 1). This is the first example of the occurrence of a 3,4-seco-lupane type triterpene glycoside in nature, as was reported in a communication to the editors of Chem. Pharm. Bull. ${ }^{1)}$ The present paper presents the experimental details of this study, including the preparation of 3,4-seco-betulinic acid derivatives (Chart 2) for use as model compounds in the structure determination of 1 . The isolation and structure determination of additional new glycosides ( 2 and 3 ) from the leaves of this plant are also described.

A suspension of a methanolic extract of the leaves collected in Korea was washed with ether and then extracted with 1-butanol saturated with water to give a glycoside mixture, which was subjected to repeated chromatography, affording 1 and two new glycosides ( 2 and 3 ) in yields of $0.04,0.05$ and $0.01 \%$, respectively. The structure determination of 1 by means of physical and chemical procedures has already been reported. ${ }^{1)}$

On hydrolysis with crude hesperidinase, ${ }^{2)} 2$ afforded an aglycone (4). The proton and carbon-13 nuclear magnetic resonance $\left({ }^{1} \mathrm{H}\right.$ - and ${ }^{13} \mathrm{C}$-NMR) spectra indicated the presence of one terminal methylene, two carbonyl and six methyl groups. On treatment with diazomethane in ether, 4 afforded a dimethyl ester (5).

In the previous communication, ${ }^{1)}$ it was reported that on acid hydrolysis with $1.5 \%$ $\mathrm{H}_{2} \mathrm{SO}_{4}$ in an aprotic solvent, a mixture of dichloromethane $\left(\mathrm{CH}_{2} \mathrm{Cl}_{2}\right)$ and dimethyl sulfoxide (DMSO), 1 gave an aglycone, named anhydro-chiisanogenin (6); an artifact formed from the genuine aglycone, chiisanogenin (7), together with several other minor products. The structure having a 2,2-dimethyltetrahydrofuran ring was proposed for 6 on the basis of ${ }^{1} \mathrm{H}$ and ${ }^{13} \mathrm{C}$-NMR spectrometry as shown in Chart 1 . Further examination of the acid hydrolysis 


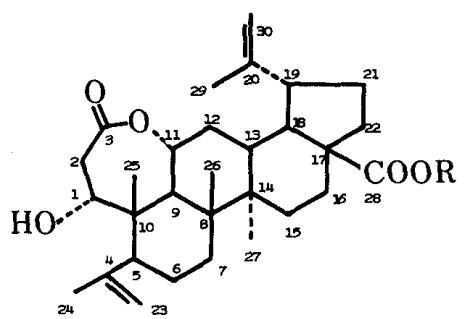

$1: \mathrm{R}=-\beta-\mathrm{Glc}^{-}-\beta-\mathrm{Glc}^{-}-\alpha-\mathrm{Rha}$

$7: \mathrm{R}=-\mathrm{H}$

$9: \mathrm{R}=-\mathrm{CH}_{3}$

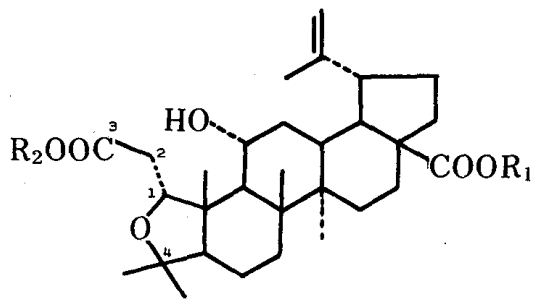

$2: \mathrm{R}_{1}=-\beta-\mathrm{Glc}^{-6} \beta-\mathrm{Glc}^{\frac{4}{4}} \alpha-\mathrm{Rha} \quad \mathrm{R}_{2}=-\mathrm{H}$

$3: \mathrm{R}_{1}=-\beta-\mathrm{Glc}^{6} \beta-\mathrm{Glc}^{4} \alpha-\mathrm{Rha} \quad \mathrm{R}_{2}=-\mathrm{CH}_{3}$

$4: \mathrm{R}_{1}=-\mathrm{H}$

$5: \mathrm{R}_{1}=-\mathrm{CH}_{3}$

$8: \mathrm{R}_{\mathrm{I}}=-\mathrm{H}$

$\mathrm{R}_{2}=-\mathrm{H}$

$\mathrm{R}_{2}=-\mathrm{CH}_{3}$

$\mathrm{R}_{2}=-\mathrm{CH}_{2} \mathrm{CH}_{3}$

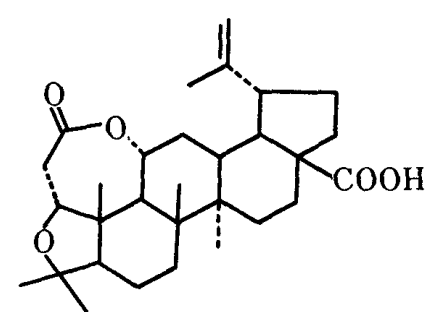

6

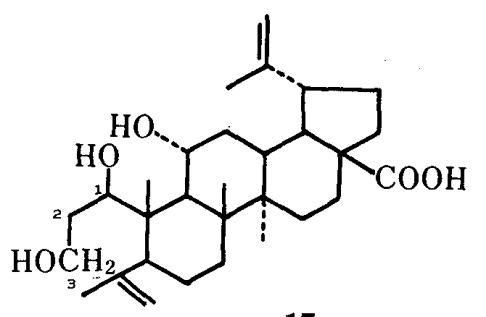

17

Chart 1

of 1 disclosed that on acid treatment with $1.5 \% \mathrm{H}_{2} \mathrm{SO}_{4}$ using $50 \%$ ethanol as a solvent, instead of $\mathrm{CH}_{2} \mathrm{Cl}_{2}-\mathrm{DMSO}, 1$ afforded not 6, but 8: On the basis of a comparison of the NMR spectral data with those of 6,8 was formulated as an ethyl ester of a hydroxy-acid formed from the lactone (6) (Chart 1). A comparison of the ${ }^{13} \mathrm{C}-\mathrm{NMR}$ spectra showed that, on going from 8 to 4, the signal due to $\mathrm{C}-3$ was shifted by $+2.4 \mathrm{ppm}$, while other carbon resonances of both compounds appeared at the same positions except for the additional signals due to the carboethoxyl group of $\mathbf{8}$. Based on these results, $\mathbf{4}$ was concluded to have the structure illustrated in Chart 1 ; it is a hydroxy-acid of 6 , and was named anhydro-chiisanogenoic acid.

The ${ }^{1} \mathrm{H}$ - and ${ }^{13} \mathrm{C}-\mathrm{NMR}$ spectra of 2 showed the presence of three monosaccharides units. Acid hydrolysis of 2 gave D-glucose and L-rhamnose. The absolute configurations of both monosaccharides were confirmed by the procedure reported by Oshima $e t$ al. ${ }^{3)}$ The electron impact mass spectrum (EI-MS) of a peracetate of 2 exhibited fragment ions at $m / z 849$ ((GlcGlc-Rha)Ac $\left.c_{9}\right), 561$ ((Glc-Rha)Ac $\left.c_{6}\right)$ and 273 ((Rha)Ac $)$. The ${ }^{13} \mathrm{C}-\mathrm{NMR}$ signals due to the sugar moiety of $\mathbf{2}$ were observed at almost the same positions as those of $\mathbf{1}$. It follows that 2 can be formulated as the $\alpha$-L-rhamnopyranosyl $(1 \rightarrow 4)-\beta$-D-glucopyranosyl $(1 \rightarrow 6)-\beta$-Dglucopyranosyl ester of $\mathbf{4}$; this compound was named isochiisanoside. Finally, the structure of $\mathbf{2}$ was confirmed by conversion of 1 into 2 under mile acidic conditions.

Glycoside 3 was obtained as a white powder. The ${ }^{1} \mathrm{H}$ - and ${ }^{13} \mathrm{C}-\mathrm{NMR}$ spectra of 3 are essentially similar to those of 2 except for the presence of signals due to a carbomethoxyl group (proton signal at $\delta 3.60(3 \mathrm{H}, \mathrm{s}$,) and carbon signals at $\delta 51.2$ and 173.4) of 3 . This indicates that $\mathbf{3}$ is a methyl ester of $\mathbf{2}$. On treatment with diazomethane, $\mathbf{2}$ afforded a methyl ester which was proved to be identical with 3 .

The possibility that 2 and $\mathbf{3}$ might be artifacts formed from 1 during the process of extraction can not be completely excluded.

\section{Experimental}

General Procedure-Melting points were determined on a Yanaco micro hot stage and are uncorrected. Optical rotations were measured with a Union PM-101 automatic digital polarimeter. Infrared (IR) spectra were 
TABLE I. ${ }^{13} \mathrm{C}$-Chemical Shifts $(\delta)$ in $\mathrm{C}_{5} \mathrm{D}_{5} \mathrm{~N}$ (Parentheses in $\mathrm{CDCl}_{3}$ )

\begin{tabular}{|c|c|c|c|c|c|c|c|c|}
\hline & 1 & 2 & 3 & 7 & & 4 & 6 & 8 \\
\hline \multicolumn{9}{|c|}{ Aglycone moiety } \\
\hline 1 & 75.0 & 87.4 & 87.1 & 75.3 & 87.5 & $(85.9)$ & $(86.4)$ & 87.2 \\
\hline 2 & 38.6 & 36.8 & 36.7 & 38.5 & 37.0 & $(36.8)$ & (37.9) & 37.0 \\
\hline 3 & 173.1 & 175.5 & 173.4 & 172.8 & 175.4 & (177.3) & (177.3) & 173.0 \\
\hline 4 & 147.6 & 79.1 & 79.3 & 147.7 & 79.1 & $(80.7)$ & $(79.7)$ & 79.2 \\
\hline 5 & 49.5 & 48.8 & 48.8 & 49.5 & 48.9 & $(47.9)$ & $(48.1)$ & 48.9 \\
\hline 6 & 25.1 & 18.7 & 18.7 & 25.1 & 18.8 & $(19.0)$ & e) & 18.8 \\
\hline 7 & 33.4 & 35.5 & 35.4 & 33.5 & 35.6 & $(35.1)$ & $(36.1)$ & 35.6 \\
\hline 8 & 41.6 & 42.7 & 42.7 & 41.6 & 42.7 & $(42.4)$ & e) & 42.7 \\
\hline 9 & 44.0 & 56.9 & 56.9 & 44.1 & 56.2 & $(55.6)$ & $(55.6)$ & 56.0 \\
\hline 10 & 44.0 & 46.8 & 46.8 & 43.9 & 46.9 & $(46.4)$ & $(46.5)$ & 46.9 \\
\hline 11 & 70.2 & 67.6 & 67.5 & 70.5 & 67.7 & $(68.1)$ & (68.3) & 67.6 \\
\hline 12 & 32.2 & 38.9 & 38.5 & 32.5 & 38.9 & (37.3) & $(35.2)$ & 38.7 \\
\hline 13 & 35.2 & 37.5 & 37.4 & 35.3 & 37.7 & $(37.1)$ & $(37.0)$ & 37.7 \\
\hline 14 & 42.1 & 42.7 & 42.7 & 42.3 & 42.9 & $(42.4)$ & $(42.5)$ & 42.9 \\
\hline 15 & 30.7 & 30.5 & 30.3 & 30.9 & 30.5 & $(30.6)$ & $(30.5)$ & 30.5 \\
\hline 16 & 32.5 & 32.2 & 32.3 & 32.6 & 32.8 & (32.3) & $(32.2)$ & 32.6 \\
\hline 17 & 56.7 & 56.1 & 56.0 & 56.3 & 56.6 & $(56.3)$ & $(56.3)$ & 56.6 \\
\hline 18 & 47.5 & 47.2 & 47.2 & 47.8 & 47.6 & $(46.8)$ & $(46.8)$ & 47.6 \\
\hline 19 & 49.5 & 49.8 & 49.4 & 49.5 & 49.5 & $(48.7)$ & $(48.8)$ & 49.5 \\
\hline 20 & 150.7 & 150.6 & 150.7 & 150.5 & 150.9 & (149.8) & (149.9) & 150.9 \\
\hline 21 & 29.5 & 30.3 & 30.8 & 29.6 & 31.2 & $(29.7)$ & (29.9) & 31.2 \\
\hline 22 & 36.8 & 36.8 & 36.9 & 37.3 & 37.4 & (37.3) & $(37.4)$ & 37.4 \\
\hline 23 & 113.9 & $24.9^{b)}$ & $24.9^{b)}$ & 113.9 & 25.0 & $(24.4)^{b)}$ & $(24.4)^{b j}$ & $24.9^{b)}$ \\
\hline 24 & 23.5 & $32.8^{b)}$ & $32.6^{b)}$ & 23.5 & 32.8 & $(32.4)^{b\rangle}$ & $(32.3)^{b)}$ & $32.8^{b)}$ \\
\hline 25 & $18.9^{a)}$ & $19.2^{a)}$ & $19.1^{a)}$ & $18.9^{a)}$ & 19.3 & $(18.7)^{a)}$ & $(18.5)^{a)}$ & $19.2^{a)}$ \\
\hline 26 & $17.9^{a)}$ & $17.8^{a)}$ & $17.8^{a)}$ & $17.8^{a)}$ & 17.9 & $(17.5)^{a)}$ & $(17.6)^{a)}$ & $17.9^{a)}$ \\
\hline 27 & 13.8 & 15.1 & 15.1 & 13.7 & 15.2 & (14.9) & $(15.0)$ & 15.2 \\
\hline 28 & 175.0 & 174.8 & 174.8 & 178.7 & 178.8 & (180.6) & (179.6) & 178.8 \\
\hline 29 & 18.9 & 19.4 & 19.5 & 18.9 & 19.5 & $(19.4)$ & $(18.9)$ & 19.6 \\
\hline 30 & 110.6 & 110.2 & 110.2 & 110.6 & 110.1 & (110.4) & $(110.2)$ & 110.1 \\
\hline$-\mathrm{OCH}_{3}$ & & & 51.2 & & & & & \\
\hline$-\mathrm{OCH}_{2} \mathrm{CH}_{3}$ & & & & & & & & 14.5 \\
\hline$-\mathrm{OCH}_{2} \mathrm{CH}_{3}$ & & & & & & & & 59.9 \\
\hline \multicolumn{9}{|l|}{ Sugar moiety } \\
\hline Glc-1 & 95.2 & 95.2 & 95.2 & & & & & \\
\hline-2 & 73.8 & 73.9 & 73.8 & & & & & \\
\hline-3 & 78.4 & $78.5^{c)}$ & $78.5^{c)}$ & & & & & \\
\hline-4 & 70.5 & 70.6 & 70.7 & & & & & \\
\hline-5 & 76.3 & 76.3 & 76.3 & & & & & \\
\hline-6 & 69.2 & 69.3 & 69.3 & & & & & \\
\hline Glc-1' & 104.7 & 104.8 & 104.9 & & & & & \\
\hline$-2^{\prime}$ & 75.2 & 75.2 & 75.2 & & & & & \\
\hline$-3^{\prime}$ & 76.9 & 76.9 & 77.0 & & & & & \\
\hline$-4^{\prime}$ & 78.4 & $78.2^{c)}$ & $78.2^{c)}$ & & & & & \\
\hline$-5^{\prime}$ & 77.8 & 77.9 & 77.9 & & & & & \\
\hline$-6^{\prime}$ & 61.3 & 61.2 & 61.2 & & & & & \\
\hline Rha-1 & 102.5 & 102.5 & 102.5 & & & & & \\
\hline-2 & $72.5^{b)}$ & $72.5^{d)}$ & $72.6^{d)}$ & & & & & \\
\hline-3 & $72.3^{b)}$ & $72.4^{d)}$ & $72.4^{d)}$ & & & & & \\
\hline-4 & 73.8 & 73.9 & 73.8 & & & & & \\
\hline-5 & 70.2 & 70.2 & 70.2 & & & & & \\
\hline-6 & 18.3 & 18.4 & 18.4 & & & & & \\
\hline
\end{tabular}

$a-d)$ These assignments may be interchanged in each column; Glc, $\beta$-D-glucopyranosyl; Rha, $\alpha$-L-rhamnopyranosyl. e) Obscure. 
taken on a Shimadzu IR-408 spectrometer. NMR spectra were recorded on a JEOL FX-100, GX-270, or FX-400 spectrometer using tetramethylsilane (TMS) as an internal standard. For gas liquid chromatography (GLC), a Shimadzu GC-8A or GC-6A was used. MS were taken on a JEOL JMS-01-SG-2 or JMS-DX-300 spectrometer by the direct inlet method; ionization voltage 75 or $70 \mathrm{eV}$. For column chromatography, Kieselgel 60 (70-230 mesh, Merck), LiChroprep RP-8 (40-63 $\mu$ m, Merck) and Diaion HP-20 (Mitsubishi Chem. Ind. Co., Ltd.) were used. All solvent systems for chromatography were homogeneous.

Acetylation for MS: A solution of a few milligram of glycoside in 5 drops of $\mathrm{C}_{5} \mathrm{H}_{5} \mathrm{~N}$ and $\mathrm{Ac}_{2} \mathrm{O}$ was allowed to stand for $24 \mathrm{~h}$ at room temperature. The reaction mixture was concentrated to dryness by blowing $\mathrm{N}_{2}$ gas over it, and the residue was subjected to MS.

Acid Hydrolysis of Glycosides and Identification ${ }^{3)}$ of Resulting Monosaccharides: A sample of glycoside $(10 \mathrm{mg})$ was heated with $3.5 \% \mathrm{HCl}$ in $\mathrm{H}_{2} \mathrm{O}$-dioxane $(1: 1)(1 \mathrm{ml})$ in a sealed microtube at $80^{\circ} \mathrm{C}$ for $3 \mathrm{~h}$. The reaction mixture was diluted with $\mathrm{H}_{2} \mathrm{O}$ and then washed with $\mathrm{CHCl}_{3}$. The aqueous layer was neutralized with Amberlite MB-3 ionexchange resin and then concentrated to give a sugar fraction. A solution of the sugar fraction ( $1 \mathrm{mg})$ in $50 \mu \mathrm{lof} \mathrm{H}_{2} \mathrm{O}$ was treated with a solution of $\alpha$-methylbenzylamine $(9 \mathrm{mg})$ and $\mathrm{NaBH}_{3} \mathrm{CN}(0.6 \mathrm{mg})$ in $50 \mu \mathrm{l}$ of EtOH, and the mixture was kept at $40^{\circ} \mathrm{C}$ for $4 \mathrm{~h}$. Then several drops of acetic acid were added, and the whole was concentrated to dryness. The residue was heated with several drops of $N$-trimethylsilylimidazole in a sealed microtube at $80^{\circ} \mathrm{C}$ for $30 \mathrm{~min}$. The reaction mixture was diluted with $\mathrm{H}_{2} \mathrm{O}$ and then extracted with $n-\mathrm{C}_{6} \mathrm{H}_{14}$. The hexane layer was washed with $\mathrm{H}_{2} \mathrm{O}$ and concentrated to dryness. A solution of the residue in $n-\mathrm{C}_{6} \mathrm{H}_{14}$ was subjected to GLC analysis (dual flame ionization detector (FID); carrier gas, He $50 \mathrm{ml} / \mathrm{min}$; WCOT glass capillary column $(0.25 \mathrm{~mm} \times 25 \mathrm{~m})$ coated with Carbowax 20M; isothermal $150^{\circ} \mathrm{C}$; injection temperature, $190^{\circ} \mathrm{C}$ ).

Extraction and Separation of Glycosides-i) Isolation of 1 from the Stem-bark: The stem-bark $(2 \mathrm{~kg})$ was defatted with $\mathrm{Et}_{2} \mathrm{O}$ and then extracted with $\mathrm{MeOH}$. The $\mathrm{MeOH}$ extract was concentrated to dryness and the residue was partitioned between $\mathrm{H}_{2} \mathrm{O}$ and 1-BuOH. The 1-BuOH layer was concentrated to give a crude glycoside fraction, which was chromatographed on a silica-gel column $\left(\mathrm{CHCl}_{3}-\mathrm{MeOH}(10: 1),(5: 1)\right.$ and $\left.(3: 1)\right)$. Elution with $\mathrm{CHCl}_{3}-\mathrm{MeOH}(3: 1)$ afforded 1 (yield, $0.2 \%$ ).

ii) Isolation of 1,2 and 3 from the Leaves: Dried leaves $(3 \mathrm{~kg}$ ) collected in Korea were extracted with hot $\mathrm{MeOH}$, and the $\mathrm{MeOH}$ extract was concentrated to dryness. The resulting extract was suspended in $\mathrm{H}_{2} \mathrm{O}$ and then washed with $\mathrm{Et}_{2} \mathrm{O}$. The aqueous layer was extracted with water-saturated 1-BuOH to give a crude glycoside fraction (49.5 g). This fraction was chromatographed on a column of highly porous polymer (Diaion $\mathrm{HP}-20$ ) and eluted with $\mathrm{H}_{2} \mathrm{O}$, $60 \% \mathrm{MeOH}, 80 \% \mathrm{MeOH}, \mathrm{MeOH}$ and $\mathrm{Me}_{2} \mathrm{CO}$, successively. The fraction eluted with $80 \% \mathrm{MeOH}$ was subjected to chromatography on silica gel. Elution with $\mathrm{CHCl}_{3}-\mathrm{MeOH}-\mathrm{H}_{2} \mathrm{O}(10: 5: 1)$ provided three fractions (frs. $1-3$ in order of elution). Fraction 1 was subjected to chromatography on a silica gel column $\left(\mathrm{CHCl}_{3}-\mathrm{MeOH}-\mathrm{H}_{2} \mathrm{O}(10: 5: 1)\right.$ and then a reversed-phase column (LiChroprep RP-8, 70\% MeOH) to give 1 (yield: $0.04 \%$ ) and 3 (yield: $0.01 \%$ ). Fraction 3 was chromatographed on a reversed-phase column (LiChroprep RP-8, 65\% MeOH) and further purified by chromatography on a silica gel column $\left(\mathrm{CHCl}_{3}-\mathrm{MeOH}-\mathrm{H}_{2} \mathrm{O}(10: 5: 1)\right)$ to give 2 (yield: $\left.0.05 \%\right)$.

Compound 1: Colorless needles (from 1-BuOH), $\operatorname{mp} 228^{\circ} \mathrm{C},[\alpha]_{\mathrm{D}}^{14}+7.7^{\circ}(c=1.69, \mathrm{MeOH})$. Anal. Calcd for $\mathrm{C}_{48} \mathrm{H}_{74} \mathrm{O}_{19} \cdot 3 \mathrm{H}_{2} \mathrm{O}: \mathrm{C}, 57.13 ; \mathrm{H}, 7.99$. Found: C, 57.12. H, 7.98. IR (Nujol): 3450 (OH), 1750, 1710 (COOR), 1640,890 $\left(\mathrm{C}=\mathrm{CH}_{2}\right) \mathrm{cm}^{-1} \cdot{ }^{1} \mathrm{H}-\mathrm{NMR}\left(\mathrm{C}_{5} \mathrm{D}_{5} \mathrm{~N}, 100 \mathrm{MHz}\right) \delta: 5.21(1 \mathrm{H}, \mathrm{d}, J=8 \mathrm{~Hz}$, anomeric $\mathrm{H}$ of Glc' $), 5.82(1 \mathrm{H}$, s, anomeric $\mathrm{H}$ of Rha), $6.40\left(1 \mathrm{H}, \mathrm{d}, J=8 \mathrm{~Hz}\right.$, anomeric $\mathrm{H}$ of Glc). EI-MS (peracetate) $m / z: 849$ ((Glc-Glc-Rha)Ac $\left.c_{9}\right), 561$ $\left((\mathrm{Glc}-\mathrm{Rha}) \mathrm{Ac}_{6}\right), 273$ (terminal-Rha-Ac $\mathrm{c}_{3}$ ). On mineral acid hydrolysis, 1 yielded D-glucose and L-rhamnose. ${ }^{13} \mathrm{C}-$ NMR data are given in Table I.

Compound 2: A white powder, $[\alpha]_{\mathrm{D}}^{17}-12.9^{\circ}(c=1.01, \mathrm{MeOH})$. Anal. Calcd for $\mathrm{C}_{48} \mathrm{H}_{76} \mathrm{O}_{20} \cdot 5 / 2 \mathrm{H}_{2} \mathrm{O}: \mathrm{C}, 56.62$; H, 8.02. Found: C, 56.43; H, 7.94. IR (Nujol): $3300(\mathrm{OH}), 1740(\mathrm{COOR}), 1700(\mathrm{COOH}), 1640,890\left(\mathrm{C}=\mathrm{CH}_{2}\right) \mathrm{cm}^{-1}$. ${ }^{1} \mathrm{H}-\mathrm{NMR}\left(\mathrm{C}_{5} \mathrm{D}_{5} \mathrm{~N}, 100 \mathrm{MHz}\right) \delta: 4.93\left(1 \mathrm{H}, \mathrm{d}, J=6.5 \mathrm{~Hz}\right.$, anomeric $\mathrm{H}$ of $\left.\mathrm{Glc}^{\prime}\right), 5.78(1 \mathrm{H}$, s, anomeric $\mathrm{H}$ of Rha), 6.28 $(1 \mathrm{H}, \mathrm{d}, J=7 \mathrm{~Hz}$, anomeric $\mathrm{H}$ of Glc). EI-MS (peracetate) $m / z: 849$ (Glc-Glc-Rha)Ac $), 561$ ((Glc-Rha)Ac $\left.\mathrm{Ac}_{6}\right), 273$ (terminal-Rha- $\mathrm{Ac}_{3}$ ). On mineral acid hydrolysis, 2 yielded $\mathrm{D}$-glucose and L-rhamnose. ${ }^{13} \mathrm{C}-\mathrm{NMR}$ data are given in Table I.

Compound 3: A white powder, $[\alpha]_{D}^{17}-10.5^{\circ}(c=0.95, \mathrm{MeOH})$. Anal. Calcd for $\mathrm{C}_{49} \mathrm{H}_{78} \mathrm{O}_{20} \cdot \mathrm{H}_{2} \mathrm{O}: \mathrm{C}, 58.55 ; \mathrm{H}$, 8.02. Found: C, 58.49; H, 8.18. IR (Nujol): $3300(\mathrm{OH}), 1740,1720$ (COOR), $1640,880\left(\mathrm{C}=\mathrm{CH}_{2}\right) \mathrm{cm}^{-1} .{ }^{1} \mathrm{H}-\mathrm{NMR}$ $\left(\mathrm{C}_{5} \mathrm{D}_{5} \mathrm{~N}, 100 \mathrm{MHz}\right) \delta: 4.83\left(1 \mathrm{H}, \mathrm{d}, J=6.5 \mathrm{~Hz}\right.$, anomeric $\mathrm{H}$ of $\left.\mathrm{Glc}^{\prime}\right), 5.77(1 \mathrm{H}, \mathrm{s}$, anomeric $\mathrm{H}$ of $\mathrm{Rha}), 6.26(1 \mathrm{H}, \mathrm{d}$, $J=7 \mathrm{~Hz}$, anomeric $\mathrm{H}$ of Glc). EI-MS (peracetate) $m / z$ : 849 ((Glc-Glc-Rha)Ac $\left.\mathrm{A}_{10}\right), 561$ ((Glc-Rha)Ac $), 273$ (terminal$\mathrm{Rha}-\mathrm{Ac}_{3}$ ). ${ }^{13} \mathrm{C}-\mathrm{NMR}$ data are listed in Table $\mathrm{I}$.

Enzymic Hydrolysis of 1 and 2-A solution of $1(150 \mathrm{mg})$ and crude hesperidinase (150 mg, Tanabe Pharm., Co., Ltd., Osaka, Japan $)^{2)}$ in $\mathrm{H}_{2} \mathrm{O}(15 \mathrm{ml})$ was incubated at $37^{\circ} \mathrm{C}$ for $40 \mathrm{~h}$. The reaction mixture was subjected to column chromatography on Diaion $\mathrm{HP}-20\left(\mathrm{H}_{2} \mathrm{O}\right.$ and $\left.\mathrm{MeOH}\right)$. The $\mathrm{MeOH}$ eluate was concentrated to dryness, and the residue was purified by column chromatography on silica gel $\left(\mathrm{CHCl}_{3}-\mathrm{MeOH}(10: 1)\right)$ to give 7 (62 $\left.\mathrm{mg}\right)$. Compound $2(120 \mathrm{mg}$ ) was also hydrolyzed in same way, yielding 4 (yield, $43 \mathrm{mg}$ ).

Compound 7: Colorless needles (from $\mathrm{Et}_{2} \mathrm{O}$ ), $\operatorname{mp} 232-234^{\circ} \mathrm{C},[\alpha]_{\mathrm{D}}^{22}+86.4^{\circ}(c=0.66, \mathrm{MeOH})$. IR $\left(\mathrm{CHCl}_{3}\right): 3340$ $(\mathrm{OH}), 1700(\mathrm{C}=\mathrm{O}), 1640,890\left(\mathrm{C}=\mathrm{CH}_{2}\right) \mathrm{cm}^{-1}$. Anal. Calcd for $\mathrm{C}_{30} \mathrm{H}_{44} \mathrm{O}_{5}: \mathrm{C}, 74.34 ; \mathrm{H}, 9.15$. Found: $\mathrm{C}, 74.14 ; \mathrm{H}$, 9.22. ${ }^{1} \mathrm{H}-\mathrm{NMR}\left(\mathrm{CDCl}_{3}, 270 \mathrm{MHz}\right) \delta: 0.91(3 \mathrm{H}), 1.02(3 \mathrm{H}), 1.08(3 \mathrm{H})\left(\mathrm{each}\right.$ s, tert $\left.-\mathrm{CH}_{3}\right), 1.73\left(3 \mathrm{H}, \mathrm{s}, \mathrm{C}_{24}-\mathrm{CH}_{3}\right), 1.69$ 
$\left(3 \mathrm{H}, \mathrm{s}, \mathrm{C}_{29}-\mathrm{CH}_{3}\right), 2.72(1 \mathrm{H}, \mathrm{dd}, J=15,8 \mathrm{~Hz} \mathrm{C}-\mathrm{Ha}), 2.93\left(1 \mathrm{H}, \mathrm{d}, J=15 \mathrm{~Hz}, \mathrm{C}_{2}-\mathrm{Hb}\right), 2.97(1 \mathrm{H}, \mathrm{ddd}, J=11,11,4 \mathrm{~Hz}$, $\left.\mathrm{C}_{19}\right), 3.57\left(1 \mathrm{H}, \mathrm{d}, J=8 \mathrm{~Hz}, \mathrm{C}_{1}-\mathrm{H}\right), 4.53\left(1 \mathrm{H}, \mathrm{ddd}, J=8,8,8 \mathrm{~Hz}, \mathrm{C}_{11}-\mathrm{H}\right), 4.83\left(1 \mathrm{H}, \mathrm{br} \mathrm{s}, \mathrm{C}_{23^{-}}-\mathrm{Ha}\right), 4.86\left(1 \mathrm{H}, \mathrm{br} \mathrm{s}, \mathrm{C}_{23^{-}}\right.$ $\mathrm{Hb}), 4.64\left(1 \mathrm{H}\right.$, br s, $\left.\mathrm{C}_{30}-\mathrm{Ha}\right), 4.76\left(1 \mathrm{H}\right.$, br s, $\left.\mathrm{C}_{30}-\mathrm{Hb}\right)$. EI-MS m/z: $484\left(\mathrm{M}^{+}\right), 469\left(\mathrm{M}^{+}-\mathrm{CH}_{3}\right), 466\left(\mathrm{M}^{+}-\mathrm{H}_{2} \mathrm{O}\right), 41$ (base peak, isopropenyl). ${ }^{13} \mathrm{C}-\mathrm{NMR}$ data are listed in Table I.

Compound 4: A white powder, $[\alpha]_{\mathrm{D}}^{20}+49.3^{\circ}(c=0.89, \mathrm{MeOH})$. Anal. Calcd for $\mathrm{C}_{30} \mathrm{H}_{46} \mathrm{O}_{6} \cdot 1 / 2 \mathrm{H}_{2} \mathrm{O}: \mathrm{C}, 70.42 ; \mathrm{H}$, 9.26. Found: $\mathrm{C}, 70.49 ; \mathrm{H}, 9.31$. IR $\left(\mathrm{CHCl}_{3}\right): 3400(\mathrm{OH}), 1695(\mathrm{COOH}), 1640,890\left(\mathrm{C}=\mathrm{CH}_{2}\right) \mathrm{cm}^{-1}$. ${ }^{1} \mathrm{H}-\mathrm{NMR}$ $\left(\left(\mathrm{CD}_{3}\right)_{2} \mathrm{CO}, 270 \mathrm{MHz}\right) \delta: 0.99(3 \mathrm{H}), 1.08(3 \mathrm{H}), 1.09(3 \mathrm{H}), 1.19(3 \mathrm{H}), 1.24(3 \mathrm{H})\left(\right.$ each s, tert $\left.-\mathrm{CH}_{3}\right), 1.72\left(3 \mathrm{H}, \mathrm{s}, \mathrm{C}_{29^{-}}\right.$ $\left.\mathrm{CH}_{3}\right), 3.10\left(1 \mathrm{H}, \mathrm{dd}, J=14,3 \mathrm{~Hz}, \mathrm{C}_{2}-\mathrm{Ha}\right), 2.29\left(1 \mathrm{H}, \mathrm{dd}, J=11,14 \mathrm{~Hz}, \mathrm{C}_{2}-\mathrm{Hb}\right), 3.05\left(1 \mathrm{H}, \mathrm{ddd}, J=11,11,3 \mathrm{~Hz}, \mathrm{C}_{19}-\mathrm{H}\right)$, $4.25\left(1 \mathrm{H}, \mathrm{dd}, J=11,3 \mathrm{~Hz}, \mathrm{C}_{1}-\mathrm{H}\right), 3.89\left(1 \mathrm{H}, \mathrm{ddd}, J=11,11,5 \mathrm{~Hz}, \mathrm{C}_{11}-\mathrm{H}\right), 4.60\left(1 \mathrm{H}, \mathrm{br} \mathrm{s}, \mathrm{C}_{30}-\mathrm{Ha}\right), 4.73\left(1 \mathrm{H}, \mathrm{br} \mathrm{s}, \mathrm{C}_{30^{-}}\right.$ $\mathrm{Hb})$. EI-MS $m / z$ : $502\left(\mathrm{M}^{+}\right), 487\left(\mathrm{M}^{+}-\mathrm{CH}_{3}\right), 41$ (base peak, isopropenyl). ${ }^{13} \mathrm{C}-\mathrm{NMR}$ data are given in Table I.

Methylation of 4 and $7-$ Compound $7\left(76 \mathrm{mg}\right.$ ) was methylated with $\mathrm{CH}_{2} \mathrm{~N}_{2}$ in $\mathrm{Et}_{2} \mathrm{Q}$, and the usual work up gave 9 (yield, $53 \mathrm{mg}$ ). Compound $4(17 \mathrm{mg}$ ) was also methylated by a similar method to give 5 (yield, $18 \mathrm{mg}$ ).

Compound 9: Colorless needles (from benzene), $\left.\mathrm{mp} 245-247.5^{\circ} \mathrm{C},[\alpha]_{\mathrm{D}}^{20}+84.0^{\circ}(c=0.5, \mathrm{MeOH}) . \mathrm{IR}(\mathrm{CHCl})_{3}\right)$ $3400(\mathrm{OH}), 1720$ (COOR), 1640, $890\left(\mathrm{C}=\mathrm{CH}_{2}\right) \mathrm{cm}^{-1} .{ }^{1} \mathrm{H}-\mathrm{NMR}\left(\mathrm{CDCl}_{3}, 100 \mathrm{MHz}\right) \delta: 0.90(3 \mathrm{H}), 1.01(3 \mathrm{H}), 1.06(3 \mathrm{H})$ (each s, tert- $\left.\mathrm{CH}_{3}\right), 1.74\left(3 \mathrm{H}, \mathrm{s}, \mathrm{C}_{24}-\mathrm{CH}_{3}\right), 1.69\left(3 \mathrm{H}, \mathrm{s}, \mathrm{C}_{29}-\mathrm{CH}_{3}\right), 3.58\left(1 \mathrm{H}, \mathrm{d}, J=8 \mathrm{~Hz}, \mathrm{C}_{1}-\mathrm{H}\right), 3.67\left(3 \mathrm{H}, \mathrm{s},-\mathrm{COOCH}_{3}\right)$, $4.85\left(2 \mathrm{H}\right.$, brs, $\left.\mathrm{C}_{23}-\mathrm{H}_{2}\right), 4.63\left(1 \mathrm{H}\right.$, br s, $\left.\mathrm{C}_{30}-\mathrm{Ha}\right), 4.76\left(1 \mathrm{H}\right.$, br s, $\left.\mathrm{C}_{30}-\mathrm{Hb}\right)$.

Compound 5: A white powder, $[\alpha]_{\mathrm{D}}^{19}+44.2^{\circ}(c=0.65, \mathrm{MeOH})$. IR $\left(\mathrm{CHCl}_{3}\right): 3400(\mathrm{OH}), 1720(\mathrm{COOR}), 1640$, $890\left(\mathrm{C}=\mathrm{CH}_{2}\right) \mathrm{cm}^{-1} \cdot{ }^{1} \cdot \mathrm{H}-\mathrm{NMR}\left(\left(\mathrm{CD}_{3}\right)_{2} \mathrm{CO}, 270 \mathrm{MHz}\right) \delta: 0.96(3 \mathrm{H}), 1.04(3 \mathrm{H}), 1.09(3 \mathrm{H}), 1.17(3 \mathrm{H}), 1.28(3 \mathrm{H})(\mathrm{each} \mathrm{s}$, tert $\left.-\mathrm{CH}_{3}\right), 1.71\left(3 \mathrm{H}, \mathrm{s}, \mathrm{C}_{29}-\mathrm{CH}_{3}\right), 3.57\left(3 \mathrm{H}, \mathrm{s},-\mathrm{COOCH}_{3}\right), 3.65\left(3 \mathrm{H}, \mathrm{s},-\mathrm{COOCH}_{3}\right), 4.23\left(1 \mathrm{H}, \mathrm{dd}, J=11,3 \mathrm{~Hz}, \mathrm{C}_{1}-\mathrm{H}\right)$, $4.26\left(1 \mathrm{H}\right.$, br s, $\left.\mathrm{C}_{30}-\mathrm{Ha}\right), 4.75\left(1 \mathrm{H}, \mathrm{br} \mathrm{s}, \mathrm{C}_{30}-\mathrm{Hb}\right)$.

Acid Hydrolysis of $1-i$ ) Formation of 6 : Concentrated $\mathrm{H}_{2} \mathrm{SO}_{4}(0.3 \mathrm{ml})$ and $\mathrm{CH}_{2} \mathrm{Cl}_{2}(18 \mathrm{ml})$ were added to a solution of $1(100 \mathrm{mg})$ in DMSO $(2 \mathrm{ml})$, and the mixture was refluxed for $2 \mathrm{~h}$ at $80^{\circ} \mathrm{C}$. After cooling, the reaction mixture was washed with $\mathrm{H}_{2} \mathrm{O}$ and then the organic layer was dried and evaporated. The residue was chromatographed on a silica gel column $\left(\mathrm{CHCl}_{3}-\mathrm{MeOH}(10: 1)\right)$ to give 6 (yield, $\left.6 \mathrm{mg}\right)$. A white powder, $[\alpha]_{\mathrm{D}}^{15}+32.6^{\circ}(c=0.46$, MeOH). IR $\left(\mathrm{CHCl}_{3}\right): 1730(\mathrm{COOR}), 1690(\mathrm{COOH}), 1640,870\left(\mathrm{C}=\mathrm{CH}_{2}\right) \mathrm{cm}^{-1} .{ }^{1} \mathrm{H}-\mathrm{NMR}\left(\mathrm{CDCl}_{3}, 400 \mathrm{MHz}\right) \delta: 0.96$ $(3 \mathrm{H}), 1.02(3 \mathrm{H}), 1.11(3 \mathrm{H}), 1.18(3 \mathrm{H}), 1.25(3 \mathrm{H})\left(\mathrm{each} \mathrm{s}\right.$, tert $\left.-\mathrm{CH}_{3}\right), 1.69\left(3 \mathrm{H}, \mathrm{s}, \mathrm{C}_{29}-\mathrm{CH}_{3}\right), 2.34(1 \mathrm{H}, \mathrm{dd}, J=11,14 \mathrm{~Hz}$, $\left.\mathrm{C}_{2}-\mathrm{Ha}\right), 3.09\left(1 \mathrm{H}, \mathrm{dd}, J=14,3 \mathrm{~Hz}, \mathrm{C}_{2}-\mathrm{Hb}\right), 3.00\left(1 \mathrm{H}\right.$, ddd, $\left.J=11,11,4 \mathrm{~Hz}, \mathrm{C}_{11}-\mathrm{H}\right), 4.31\left(1 \mathrm{H}, \mathrm{dd}, J=11,3 \mathrm{~Hz}, \mathrm{C}_{1}-\mathrm{H}\right)$, $3.94\left(1 \mathrm{H}\right.$, ddd, $\left.J=11,11,5 \mathrm{~Hz}, \mathrm{C}_{11}-\mathrm{H}\right), 4.64\left(1 \mathrm{H}, \mathrm{brs}, \mathrm{C}_{30}-\mathrm{Ha}\right), 4.76\left(1 \mathrm{H}, \mathrm{brs}, \mathrm{C}_{30}-\mathrm{Hb}\right)$. High-resolution MS $m / z$ : $484.3166\left(\mathrm{M}^{+}\right.$, Calcd for $\left.\mathrm{C}_{30} \mathrm{H}_{44} \mathrm{O}_{5}: 484.3189\right) .{ }^{13} \mathrm{C}-\mathrm{NMR}$ data are given in Table $\mathrm{I}$.

ii) Formation of 8: A solution of $1(90 \mathrm{mg})$ in $1.5 \% \mathrm{H}_{2} \mathrm{SO}_{4} / \mathrm{H}_{2} \mathrm{O}-\mathrm{EtOH}(1: 1)(20 \mathrm{ml})$ was refluxed for $4 \mathrm{~h}$ and the reaction mixture was neutralized with Amberlite MB-3 resin and then concentrated to dryness. The crude product was subjected to chromatography on silica gel $\left(\mathrm{CHCl}_{3}-\mathrm{MeOH}-\mathrm{H}_{2} \mathrm{O}(10: 5: 1)\right.$ ) to give 8 (yield, $13 \mathrm{mg}$ ). A white powder, $[\alpha]_{\mathrm{D}}^{19}+40.3^{\circ}(c=0.90, \mathrm{MeOH})$. IR $\left(\mathrm{CHCl}_{3}\right)$ : $1720(\mathrm{COOR}), 1690(\mathrm{COOH}), 1640,870\left(\mathrm{C}=\mathrm{CH}_{2}\right) \mathrm{cm}^{-1} \cdot{ }^{1} \mathrm{H}$ $\operatorname{NMR}\left(\mathrm{C}_{5} \mathrm{D}_{5} \mathrm{~N}, 100 \mathrm{MHz}\right) \delta: 1.11\left(3 \mathrm{H}, \mathrm{t}, J=6 \mathrm{~Hz}, \mathrm{CH}_{3} \mathrm{CH}_{2}-\right), 1.12(3 \mathrm{H}), 1.16(3 \mathrm{H}), 1.32(3 \mathrm{H}), 1.40(3 \mathrm{H})$ (each s, tert$\left.\mathrm{CH}_{3}\right), 1.76\left(3 \mathrm{H}, \mathrm{s}, \mathrm{C}_{29}-\mathrm{CH}_{3}\right), 3.84\left(2 \mathrm{H}, \mathrm{q}, J=6 \mathrm{~Hz}, \mathrm{CH}_{3} \mathrm{CH}_{2} \mathrm{O}-\right), 4.16\left(1 \mathrm{H}, \mathrm{dd}, J=12,6 \mathrm{~Hz}, \mathrm{C}{ }^{-} \mathrm{H}\right) .4 .68\left(1 \mathrm{H}, \mathrm{br} \mathrm{s}, \mathrm{C}_{30}-\right.$ $\mathrm{Ha}), 4.84\left(1 \mathrm{H}\right.$, brs, $\left.\mathrm{C}_{30}-\mathrm{Hb}\right) .{ }^{13} \mathrm{C}-\mathrm{NMR}$ data are given in Table $\mathrm{I}$.

Reductive Lactone Ring Cleavage ${ }^{4)}$ of $7-\mathrm{A}$ solution of $7(40 \mathrm{mg}), \mathrm{NaBH}_{4}(5.8 \mathrm{mg})$ and $\mathrm{AlCl}_{3}(20 \mathrm{mg})$ in diglyme $(4 \mathrm{ml})$ was heated for $2 \mathrm{~h}$ at $70^{\circ} \mathrm{C}$. After cooling, the reaction mixture was poured into $2 \mathrm{~N} \mathrm{HCl}(50 \mathrm{ml})$ and the whole was extracted with $\mathrm{CHCl}_{3}$. The organic layer was washed with $\mathrm{H}_{2} \mathrm{O}$, dried and evaporated to dryness. The residue was purified by silica gel column chromatography $\left(\mathrm{CHCl}_{3}-\mathrm{MeOH}(10: 1)\right)$ to give 17 (yield, $\left.18 \mathrm{mg}\right)$. A white powder, $[\alpha]_{\mathrm{D}}^{16}+40.0^{\circ}(c=0.30, \mathrm{MeOH}) .{ }^{1} \mathrm{H}-\mathrm{NMR}\left(\mathrm{CDCl}_{3}, 270 \mathrm{MHz}\right) \delta: 1.01(3 \mathrm{H}), 1.03(3 \mathrm{H}), 1.31(3 \mathrm{H})($ each s, tert$\left.\mathrm{CH}_{3}\right), 1.84\left(3 \mathrm{H}, \mathrm{s}, \mathrm{C}_{24}-\mathrm{CH}_{3}\right), 1.70\left(3 \mathrm{H}, \mathrm{s}, \mathrm{C}_{29}-\mathrm{CH}_{3}\right), 3.00\left(1 \mathrm{H}, \mathrm{ddd}, J=11,11,4 \mathrm{~Hz}, \mathrm{C}_{19}-\mathrm{H}\right), 3.97(1 \mathrm{H}, \mathrm{ddd}, J=11,11$, $\left.6 \mathrm{~Hz}, \mathrm{C}_{11}-\mathrm{H}\right), 4.76\left(1 \mathrm{H} \times 2\right.$, br s, $\mathrm{C}_{23}-\mathrm{Ha}$ and $\left.\mathrm{C}_{23}-\mathrm{Hb}\right), 4.88\left(1 \mathrm{H}, \mathrm{br} \mathrm{s}, \mathrm{C}_{30}-\mathrm{Hb}\right), 4.63\left(1 \mathrm{H}\right.$, br s, $\left.\mathrm{C}_{30}-\mathrm{Ha}\right)$. High-resolution MS $m / z$ : $488.3512\left(\mathrm{M}^{+}\right.$, Calcd for $\left.\mathrm{C}_{30} \mathrm{H}_{48} \mathrm{O}_{5}: 488.3502\right)$.

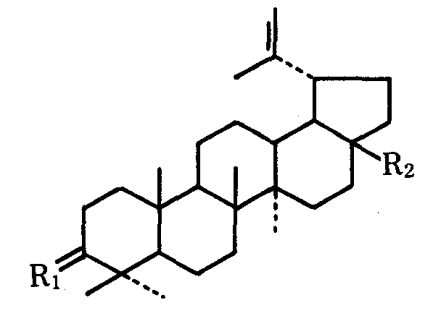

$\begin{array}{ll}10: \mathrm{R}_{1}=-\mathrm{OH} & \mathrm{R}_{2}=-\mathrm{CH}_{2} \mathrm{OH} \\ 11: \mathrm{R}_{1}==\mathrm{O} & \mathrm{R}_{2}=-\mathrm{COOH} \\ 12: \mathrm{R}_{1}==\mathrm{NOH} & \mathrm{R}_{2}=-\mathrm{COOH}\end{array}$

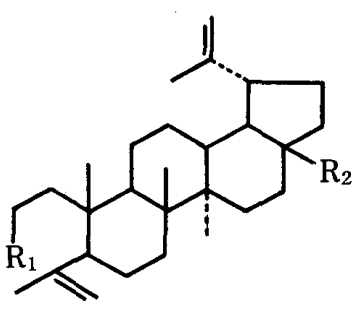

$13: \mathrm{R}_{1}=-\mathrm{CN}$
$14: \mathrm{R}_{1}=-\mathrm{COOH}$
$15: \mathrm{R}_{1}=-\mathrm{COOCH}_{3}$
$16: \mathrm{R}_{1}=-\mathrm{CH}_{2} \mathrm{OH}$

$\mathrm{R}_{2}=-\mathrm{COOH}$

$\mathrm{R}_{2}=-\mathrm{COOH}$

$\mathrm{R}_{2}=-\mathrm{COOCH}_{3}$

$\mathrm{R}_{2}=-\mathrm{CH}_{2} \mathrm{OH}$ 
Synthesis of 16-i) Cleavage of Ring A of 11 (Abnormal Beckmann Rearrangement): According to the reported procedure, ${ }^{5)}$ a solution of $11(455 \mathrm{mg})$, derived from betulin (10), ${ }^{6)} \mathrm{CH}_{3} \mathrm{COONa}(160 \mathrm{mg})$ and $\mathrm{NH}_{2} \mathrm{OH} \cdot \mathrm{HCl}$ $(100 \mathrm{mg})$ in $\mathrm{EtOH}(50 \mathrm{ml})$ was refluxed for $20 \mathrm{~h}$. The reaction mixture was diluted with $\mathrm{H}_{2} \mathrm{O}$ and then extracted with $\mathrm{Et}_{2} \mathrm{O}$. The organic layer was subjected to silica gel column chromatography $\left(\mathrm{CHCl}_{3}\right)$ to give $12(438 \mathrm{mg})$. A mixture of $12(438 \mathrm{mg})$ and $p$-toluenesulfonyl chloride $(1 \mathrm{~g})$ in dry pyridine $(20 \mathrm{ml})$ was refluxed for $17 \mathrm{~h}$ under an $\mathrm{N}_{2}$ stream. The reaction mixture was poured into $3 \mathrm{~N} \mathrm{HCl}(50 \mathrm{ml})$ and then extracted with $\mathrm{Et}_{2} \mathrm{O}$. The organic layer was concentrated to dryness and the residue was chromatographed on silica gel $\left(\mathrm{CHCl}_{3}\right)$ to afford $13(375 \mathrm{mg})$.

ii) Hydrolysis of 13: A solution of $13(375 \mathrm{mg})$ in $20 \% \mathrm{KOH}-\mathrm{EtOH}(10 \mathrm{ml})$ was refluxed for $3 \mathrm{~h}$. The reaction mixture was neutralized with Amberlite MB-3 resin and concentrated to dryness. The residue was purified by silica gel chromatography $\left(\mathrm{CHCl}_{3}-\mathrm{MeOH}(10: 1)\right)$ to give $14(202 \mathrm{mg})$.

iii) Methylation of 14: Compound 14 was methylated with $\mathrm{CH}_{2} \mathrm{~N}_{2}$ in $\mathrm{Et}_{2} \mathrm{O}$ and after work up as usual, the product was subjected to silica gel chromatography $\left(\mathrm{CHCl}_{3}\right)$ to give $15(198 \mathrm{mg})$. Colorless syrup, $[\alpha]_{\mathrm{D}}^{24}+15.2^{\circ}$ $(c=3.0, \mathrm{MeOH})$. IR $\left(\mathrm{CHCl}_{3}\right): 1720\left(\mathrm{COOCH}_{3}\right), 1635,890\left(\mathrm{C}=\mathrm{CH}_{2}\right) \mathrm{cm}^{-1} .{ }^{1} \mathrm{H}-\mathrm{NMR}\left(\mathrm{CDCl}_{3}, 100 \mathrm{MHz}\right) \delta: 0.82$ $(3 \mathrm{H}), 0.96(3 \mathrm{H}), 0.98(3 \mathrm{H}), 1.70(3 \mathrm{H}), 1.72(3 \mathrm{H})\left(\right.$ each s, tert $\left.-\mathrm{CH}_{3}\right), 3.65(3 \mathrm{H}), 3.67(3 \mathrm{H})\left(\mathrm{each} \mathrm{s},-\mathrm{COOCH}_{3}\right), 4.84(1 \mathrm{H}$, br s, $\left.\mathrm{C}_{23}-\mathrm{Ha}\right), 4.63\left(1 \mathrm{H} \times 2\right.$, br s, $\mathrm{C}_{23}-\mathrm{Hb}$ and $\left.\mathrm{C}_{30}-\mathrm{Ha}\right), 4.73\left(1 \mathrm{H}\right.$, br s, $\left.\mathrm{C}_{30}-\mathrm{Hb}\right)$.

iv) Reduction of 15: A solution of $15(160 \mathrm{mg})$ and $\mathrm{LiAlH}_{4}(100 \mathrm{mg})$ in anhydrous $\mathrm{Et}_{2} \mathrm{O}(20 \mathrm{ml})$ was allowed to stand for $24 \mathrm{~h}$ at room temperature. The reaction mixture was acidified with $10 \% \mathrm{H}_{2} \mathrm{SO}_{4}$ and extracted with $\mathrm{Et}_{2} \mathrm{O}$. The $\mathrm{Et}_{2} \mathrm{O}$ layer was washed with $\mathrm{H}_{2} \mathrm{O}$, dried over anhydrous $\mathrm{Na}_{2} \mathrm{SO}_{4}$ and evaporated to dryness. The residue $(148 \mathrm{mg})$ was crystallized from benzene to give $16(84 \mathrm{mg})$. Colorless needles, $\mathrm{mp} 212-213^{\circ} \mathrm{C},[\alpha]_{\mathrm{D}}^{22}+45.3^{\circ}(c=1.78$, $\mathrm{MeOH})$. Anal. Calcd for $\mathrm{C}_{30} \mathrm{H}_{50} \mathrm{O}_{2}: \mathrm{C}, 81.39 ; \mathrm{H}, 11.38$. Found: $\mathrm{C}, 81.10 ; \mathrm{H}, 11.58$. IR $\left(\mathrm{CHCl}_{3}\right): 3400(\mathrm{OH}), 1640,885$ $\left(\mathrm{C}=\mathrm{CH}_{2}\right) \mathrm{cm}^{-1} .{ }^{1} \mathrm{H}-\mathrm{NMR}\left(\mathrm{CDCl}_{3}, 100 \mathrm{MHz}\right) \delta: 0.80(3 \mathrm{H}), 1.01(3 \mathrm{H}), 1.07(3 \mathrm{H}), 1.70(3 \mathrm{H} \times 2)\left(\right.$ each s, tert $\left.-\mathrm{CH}_{3}\right)$, $3.56\left(2 \mathrm{H}, \mathrm{t}, J=8 \mathrm{~Hz}, \mathrm{C}_{3}-\mathrm{H}_{2}\right), 3.33\left(1 \mathrm{H}, \mathrm{d}, J=11 \mathrm{~Hz}, \mathrm{C}_{28}-\mathrm{Ha}\right), 3.80\left(1 \mathrm{H}, \mathrm{d}, J=11 \mathrm{~Hz}, \mathrm{C}_{28}-\mathrm{Hb}\right), 4.60\left(1 \mathrm{H} \times 2, \mathrm{br} \mathrm{s}, \mathrm{C}_{23}-\right.$ $\mathrm{Hb}$ and $\left.\mathrm{C}_{30}-\mathrm{Ha}\right), 4.68\left(1 \mathrm{H}\right.$, brs, $\left.\mathrm{C}_{30}-\mathrm{Hb}\right), 4.81\left(1 \mathrm{H}, \mathrm{brs}, \mathrm{C}_{23}-\mathrm{Ha}\right)$.

Modified Horeau's Method ${ }^{73}$ for 7 (Determination of Chirality of C-1)—A solution of 7 (3 mg) and ( \pm$)-2-\alpha-$ phenylbutyric acid anhydride $(1.8 \mathrm{mg})$ in dry pyridine was allowed to stand in a sealed micro tube at room temperature for $20 \mathrm{~h}$, then $12 \mu \mathrm{l}$ of $(+)-(R)-\alpha$-phenylethylamine was added. After standing for $30 \mathrm{~min}$, then mixture was concentrated to dryness by blowing $\mathrm{N}_{2}$ gas over it. The residue extracted with a small amount of EtOAc and the solution was subjected to GLC analysis (dual FID; carrier gas, $\mathrm{N}_{2} 2.1 \mathrm{~kg} / \mathrm{cm}^{2}$; column packed with $2 \% \mathrm{SE}-30$, $2 \mathrm{~m} \times 2.6 \mathrm{~mm}$; isothermal $200^{\circ} \mathrm{C}$; injection and detector temperature, $250{ }^{\circ} \mathrm{C}$ ). The relative proportions of the amides of $(-)-(R)$-and $(+)-(S)-\alpha$-phenylbutyric acid were calculated from the areas of their peaks. Subtraction of the corresponding value from the reaction with cyclohexanol gave the decrement of the percentage area representing the $(-)-(R)$-acid: $-5.9 \%$.

Cleavage of Ester-Glycoside Linkage ${ }^{8)}$ of 1 - A solution of $1(100 \mathrm{mg})$, anhydrous LiI (100 mg) and 2,6-lutidine $(5 \mathrm{ml})$ in anhydrous $\mathrm{MeOH}(5 \mathrm{ml})$ was refluxed for $15 \mathrm{~h}$. The reaction mixture was deionized with Amberlite MB-3 resin and concentrated to dryness. The residue was chromatographed on Diaion $\mathrm{HP}-20$ with $\mathrm{H}_{2} \mathrm{O}$ to give methyl oligoglycoside (yield, $23 \mathrm{mg}$ ), which was identified by comparison of the ${ }^{13} \mathrm{C}-\mathrm{NMR}$ spectrum with that of an authentic sample. ${ }^{8)}$

Formation of 2 from 1 - A solution of $1(135 \mathrm{mg})$ in $3.5 \% \mathrm{HCl} / \mathrm{H}_{2} \mathrm{O}$-dioxane $(1: 1)$ was allowed to stand for $41 \mathrm{~h}$ at room temperature. The solution was neutralized with Amberlite MB-3 resin and then concentrated to dryness. The crude product was purified by silica gel column chromatography $\left(\mathrm{CHCl}_{3}-\mathrm{MeOH}-\mathrm{H}_{2} \mathrm{O}(10: 5: 1)\right)$ to give an amorphous powder (yield, $84 \mathrm{mg}$ ), which was identified by comparison of physical constants and spectral data with those of 2.

Methylation of $2-$ Compound $2\left(37 \mathrm{mg}\right.$ ) was treated with $\mathrm{CH}_{2} \mathrm{~N}_{2}$ in $\mathrm{Et}_{2} \mathrm{O}-\mathrm{MeOH}$ to give a methyl ester (yield, $35 \mathrm{mg}$ ), which was identified by comparison of physical constants and spectral data with those of 3 .

Acknowledgement We are grateful to Dr. H. Matsuura, Wakunaga Pharm. Ind. Co., Ltd., Hiroshima for measurement of high-resolution of EI-MS and ${ }^{1} \mathrm{H}-\mathrm{NMR}$ at $270 \mathrm{MHz}$.

\section{References}

1) D.-R. Hahn, R. Kasai, J.-H. Kim, A. Taniyasu, and O. Tanaka, Chem. Pharm. Bull., 32, 1244 (1984).

2) H. Kohda and O. Tanaka, Yakugaku Zasshi, 95, 246 (1975).

3) R. Oshima, J. Kumanotani, and C. Watanabe, J. Chromatogr., 259, 159 (1983).

4) G. R. Pettit and T. R. Kasturi, J. Org. Chem., 26, 4557 (1961).

5) a) G. P. Moss and S. A. Nicolaidis, Chem. Commun., 1969, 1077; b) C. W. Shoppee, N. W. Hughes, R. E. Lack, and J. T. Pinhey, J. Chem. Soc. (C), 1970,:1433; c) K. F. Cohen, R. Kazlauskas, and J. T. Pinhey, J. Chem. Soc., Perkin Trans. 1, 1973, 2076.

6) L. Ruzicka, A. H. Lamberton, and E. W. Christie, Helv. Chim. Acta, 21, 1706 (1983).

7) C. J. W. Brooks and J. D. Gilbert, J. Chem. Soc., Chem. Commun., 1973, 197.

8) K. Ohtani, K. Mizutani, R. Kasai, and O. Tanaka, Tetrahedron Lett., 25, 4537 (1984). 